\title{
A Fuzzy Susceptible-Exposed-Infected-Recovered Model Based on the Confidence Index
}

\author{
Chenming $\mathrm{Li}^{1,3} \cdot$ Jin Huang ${ }^{4} \cdot$ Ye-Hwa Chen ${ }^{2,4} \cdot$ Han Zhao $^{1}$
}

Received: 30 March 2020/Revised: 22 November 2020/Accepted: 30 November 2020/Published online: 5 February 2021

(C) Taiwan Fuzzy Systems Association 2021

\begin{abstract}
In this paper, the susceptible-exposed-infectedrecovered (SEIR) model is applied to the novel coronavirus disease. With the actual data in Georgia, USA, we obtained the related parameters such as the recovery rate and mortality rate. Then, the development of the novel coronavirus is investigated. For more accuracy, we consider the parameters in this model as the functions of the infected number and disease duration. These parameters' functions are used to reflect the impact of disease development on parameters. Furthermore, the coefficients in these functions are regarded as uncertainties. To obtain these uncertain coefficients, the fuzzy set theory and confidence index theory are adopted. Thus, the fuzzy SEIR model is proposed.
\end{abstract}

Keywords SEIR model - Uncertain parameters · Confidence index $\cdot$ Fuzzy set theory

Ye-Hwa Chen

yehwa.chen@me.gatech.edu

1 School of Mechanical Engineering, Hefei University of Technology, Hefei 230009, Anhui, China

2 National Engineering Laboratory for Highway Maintenance Equipment, Chang'an University, Xi' an 710065, Shaanxi, China

3 The George W. Woodruff School of Mechanical Engineering, Georgia Institute of Technology, Atlanta, GA 30332, USA

4 School of Vehicle and Mobility, Tsinghua University, Beijing 100084, China

\section{Introduction}

Coronavirus (or COVID-19 virus) has become a global infectious disease and more and more people are affected. Until now, more than 44,000 people are infected and 783 people dead in the United States [1] alone. At this time, there are no specific vaccines or treatments for COVID-19. However, there are many ongoing clinical trials evaluating potential treatments. The World Health Organization (WHO) will continue to provide updated information as soon as clinical findings become available. The whole society is influenced by the spread of this disease. The schools have shut down. Many people snap up daily necessities in supermarkets out of fear of viruses. Even more serious social problems have also emerged. Thus, this kind of disease needs to be known scientifically. People should understand how the disease will develop under different measures. First, a suitable model is needed to describe the disease development. To better understand how the disease is transmitted, the SEIR model [2-4] is studied and improved in this paper.

In the Epidemiology field, the SIR model is well-known, and many great achievements have been made on that [5-7]. In this model, $S, I, R$ represent the susceptible, infected and recovered people. However, the coronavirus disease exists a latent phase during which the individual is infected but not yet symptomatic. A new part $E$ is introduced in the SEIR model to describe the non-symptomatic but infected phase.

In [8], a modified model is used to study the Ebola. The effects of hospitalization and the spread of disease through interactions with deceased, but infectious, individuals are considered. In [9], the Lyapunov function is constructed to investigate the global stability of disease-free and endemic equilibria based on the SEIR model. Recently, this model is 
also applied to predict the coronavirus spread [10] in Wuhan City, in which the Spring Festival is taken into consideration. In [11], fractional calculus is applied to the SEIR model to predict the disease development in China. The analytical solution of the SEIR model is studied for the free spread of the COVID-19 pandemic in [12]. A multistrain SEIR epidemic model is established for the COVID19 pandemic with general incidence rates in [13]. The above studies have made some great achievements in investigating the COVID-19 pandemic. Different from them, this paper focuses on the current hardest-hit area. Furthermore, the SEIR model is combined with the fuzzy set theory to show the disease development. Next, we use the confidence index theory which is based on confidence interval [14] to measure the fuzzy portions in the SEIR model. This theory was applied to many fields, such as medicine [15] and mechanical engineering [16]. In this paper, a confidence index function was designed to solve the expectation values of fuzzy numbers. More specific details are given as follows. In this paper, we endeavor to explore the spread of the disease from a fuzzy dynamic system point of view. At the outset, the development of this disease is studied based on the State of Georgia, USA, data. Two predictions are given when there is no control and under the current measures. Then, the keys parameters such as the contact and recovery rate are modified as functions of the infected number and time. This process is to reflect, on one hand, the relationship between the importance people attach to disease and the number of people infected, on the other hand, the improvement of treatment over time. Furthermore, the coefficients in the functions are considered as uncertainty. As for uncertainty, it is a non-negligible factor in an actual system. In the engineering field, many approaches have been proposed to deal with it [17-19]. Recently, fuzzy theory gets more and more attention. For example, many inspiring works have been done based on fuzzy logic theory. In [20], a novel adaptive fault-tolerant control was proposed and the fuzzy logic systems were used to deal with the unknown nonlinear faults. The authors proved that the tracking error converged to a small area around the origin and all signals within the closed-loop system remained bounded. In this paper, the fuzzy set theory is applied, which is proposed in 1965 by Zadeh [21]. Many achievements have been done based on this theory, such as decision making [22, 23], control design $[24,25]$ and optimization problem[26-28]. As a result, the uncertainty in this SEIR model is described by fuzzy numbers. Then, the confidence index theory is used to seek the expectation values of these fuzzy numbers. In this way, the parameters are able to vary their values along with the external environment. As a result, the modified fuzzy model can reflect the actual situation more precisely.
The main difficulties in this paper are finding the suitable parameters in the SEIR model based on the actual data for this novel disease and solving the expected values of the fuzzy portions in the modified model based on fuzzy set theory and confidence index theory to accurately predict disease progression.

In summary, the salient contributions of this paper are threefold. First, the disease predictions in the State of Georgia, USA, are studied under different measures. Second, the parameters in the SEIR model are regarded as functions to reflect the actual situation more accurately. Third, some uncertain coefficients are described in a fuzzy way. The fuzzy set theory and confidence index theory are applied to establish the fuzzy SEIR model.

\section{SEIR Model}

To describe the coronavirus disease, the SEIR model is adopted. Because there is a significant incubation period during which individuals have been infected but are not yet infectious themselves. The relationships are shown in Fig. 1.

The parameters' meanings in this figure are given in Table 1. Based on the relationships, consider the SEIR model [29] as

$$
\begin{aligned}
\dot{S} & =-r b S I / N-r_{2} b_{2} S E / N, \\
\dot{E} & =r b S I / N-a E+r_{2} b_{2} S E / N, \\
\dot{I} & =a E-y I-u I, \\
\dot{R} & =y I, \\
\dot{N} & =-u I, \\
\dot{D} & =u I,
\end{aligned}
$$

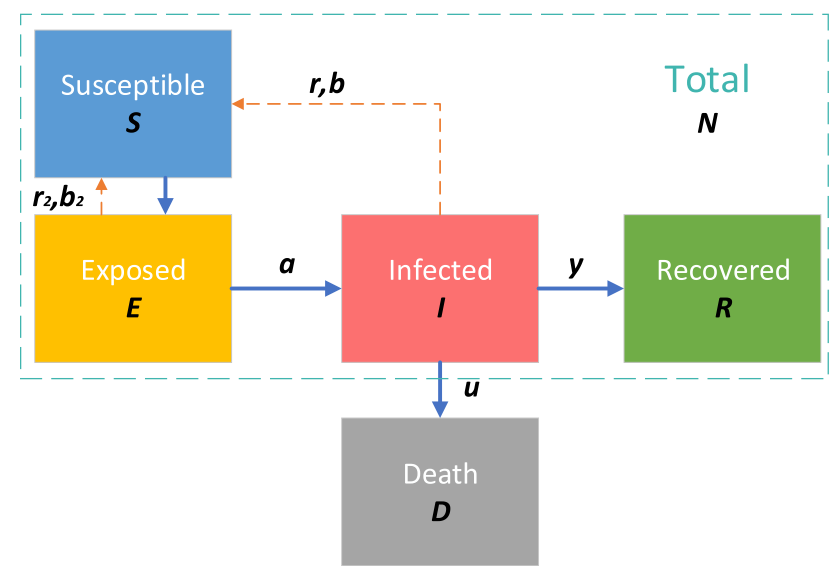

Fig. 1 Relationships between each part of the SEIR model 
Table 1 Parameters

\begin{tabular}{ll}
\hline$r$ & Number of susceptible-infected contacts \\
$r_{2}$ & Number of susceptible-exposed contacts \\
$b$ & Probability of transmitting disease between a susceptible and an infectious individual \\
$b_{2}$ & Probability of transmitting disease between a susceptible and an exposed individual \\
$a$ & Rate at which an exposed person becomes infective \\
$y$ & Recovery rate \\
$u$ & Mortality rate \\
\hline
\end{tabular}

where $S$ is the number of susceptible population, $E$ is the number of persons exposed to or in the latent period of the disease, $I$ is the number of infected persons, $R$ is the number of cases who recover, $D$ is the number of death cases, $N$ is the total population. Thus, $N=S+E+I+R-D$.

In this model, there are susceptible people and few infected people at the beginning. Then, the susceptible people may become exposed to people after contact with the infected people. The contact number and probability of transmitting disease are $r$ and $b$, respectively. The exposed people can also give the virus to the susceptible. We use $r_{2}$ and $b_{2}$ to represent the contact number and probability of transmitting disease. After the latent period, the exposed people have a chance, $a$, to become the infected people. Finally, infected people may be either recovered or died with a recovery rate $y$ and a mortality rate $u$.

\section{Data Fitting and Prediction}

We set the initial conditions as $N_{0}=100,000, E_{0}=0$, $I_{0}=2, R_{0}=0, D_{0}=0, S_{0}=99,998$. The actual data for the first 21 days are given in Table 2 [30] and Fig. 2 [1].

Table 2 shows that the growth rate decreased from the 11 th day (03-12). The reason is that some measures are adopted, such as the establishment of the quarantine destination on 03-09 [31]. Thus, the data are divided into two parts for data fitting.

Table 2 COVID-19 cases in Georgia

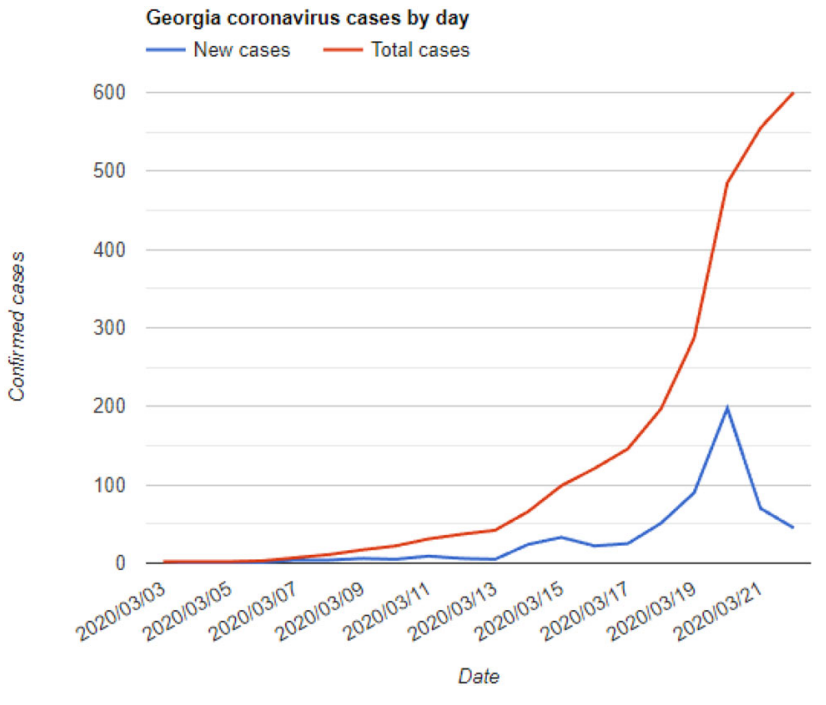

Fig. 2 Actual data from 03-02 to 03-22 in Georgia, USA

For the first part, that is from 03-02 to 03-11, we choose the relevant parameters as $r=15, b=0.045, a=0.6$, $r_{2}=25, \quad b_{2}=0.03, \quad y=0.1, u=0.034$. This set of parameters represent that the government and people take nothing measures for this virus. Fig. 3 shows the development of this disease under this circumstance.

In this figure, we set 03-02 as the first day. The red star line represents the actual infected number, which is equal to the red line in Fig. 3 from 03-02 to 03-11. From this figure, there would be almost 25,000 death cases and 55,000 infected cases. It also shows that this disease will

\begin{tabular}{llll}
\hline Date & COVID-19 cases in Georgia (growth rate) & $03-02$ & 2(n.a.) \\
\hline $03-03$ & $2(=)$ & $03-04$ & $2(=)$ \\
$03-05$ & $2(=)$ & $03-06$ & $3(+50 \%)$ \\
$03-07$ & $8(+166 \%)$ & $03-08$ & $11(+37 \%)$ \\
$03-09$ & $15(+36 \%)$ & $03-10$ & $17(+13 \%)$ \\
$03-11$ & $27(+59 \%)$ & $03-12$ & $33(+22 \%)$ \\
$03-13$ & $64(+94 \%)$ & $03-14$ & $66(+3 \%)$ \\
$03-15$ & $99(+50 \%)$ & $03-16$ & $121(+22 \%)$ \\
$03-17$ & $146(+21 \%)$ & $03-18$ & $197(+35 \%)$ \\
$03-19$ & $287(+46 \%)$ & $03-20$ & $420(+46 \%)$ \\
$03-21$ & $555(+32 \%)$ & $03-22$ & $620(+12 \%)$ \\
\hline
\end{tabular}




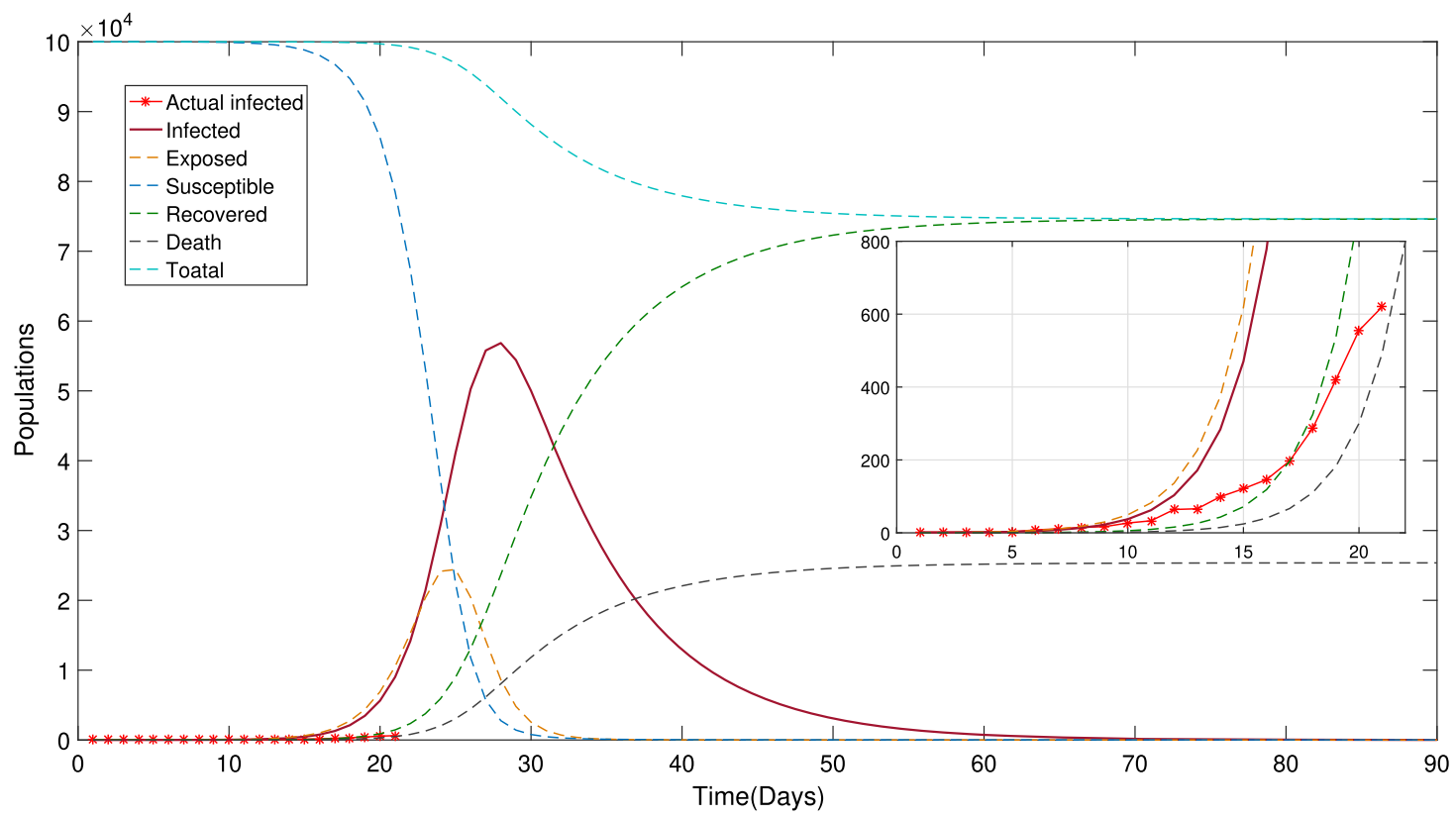

Fig. 3 Disease development without control

last for 70 days if the disease would spread without any control. However, the actual infected number is far less than the prediction after the 10th day. It appears that the disease is controlled to some degree because of the measures we adopted such as school closed.

Therefore, the parameters need to be revised to fit the second part data from 03-12 to 03-22. Based on the current measures, the relevant parameters are chosen as $r=12$, $r_{2}=20, b=0.032, b_{2}=0.025, y=0.3$. Figure 4 shows the development of this disease with the revised parameters after the 11th day.

With the given parameters $\left(r=12, r_{2}=20, b=0.032\right.$, $b_{2}=0.025, y=0.3$ after the 10th day), the simulation of the first 21 days data is coincident with the actual data. In this case, we predict that the disease may infect 15,000 and lead to 9000 death. The consequence is still serious under these current measures.

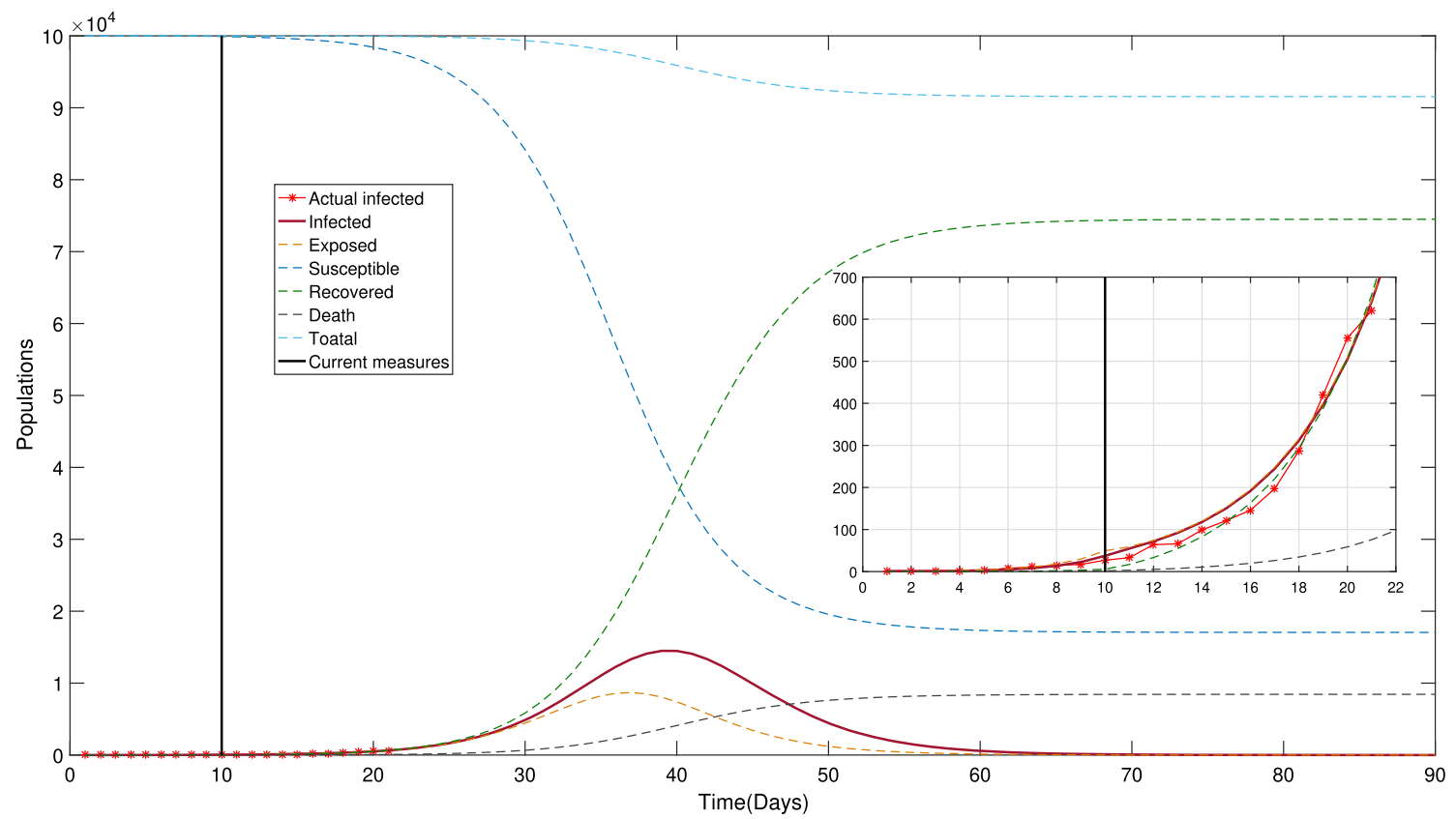

Fig. 4 Disease development based on current measures 
If the government takes more measures and people become more cautious about this disease after the 21 st day $(03-22)$, the prediction of this disease is given in Fig. 5 with $r=12, r_{2}=19, b=0.032, b_{2}=0.024, y=0.31$, $u=0.032$.

Remark 1 The manuscript was revised on 06-15. The actual data from 03-23 to 04-05 [30] are added in Fig. 5 as a comparison.

Remark 2 In this section, the disease developments are discussed in three different situations. A suitable group of related parameters is found based on the actual data. The simulations results show that the decrease of contact numbers and the probability of transmitting can lead to important influences on the number of death and disease duration. Hence, we should reduce contact with people to reduce the value of $r$, and wear masks and gloves to reduce the value of $b$. However, in an actual model, these parameters are not fixed, and it is difficult to obtain the exact values. In the next section, we will regard these parameters as the functions of the number of infected cases and time. The fuzzy theory will be applied to describe them.

\section{Parameters Functions and Fuzzy Coefficients}

In the classical SEIR model, the parameters are regarded as constants. However, they are influenced by government policies, people's attitudes to the disease and etc. In this paper, a novel method is proposed to deal with these parameters.

In the modified SEIR model, the number of susceptibleexposed contacts $r_{2}$, probability of transmitting disease between a susceptible and an exposed individual $b_{2}$, recovery rate $y$, and mortality rate $u$ are uncertain. They are considered as the functions of the number of infected cases and time, which are given as follows:

$$
\begin{aligned}
& r_{2}(I)=\phi \Delta I+c_{1}, \\
& b_{2}(I)=\psi \Delta I+c_{2}, \\
& y(T)=\mu T+c_{3}, \\
& u(T)=\rho T+c_{4},
\end{aligned}
$$

where $\Delta I=I(T)-I(T-1)$ is the growth of infected cases, $\phi, \psi, \mu$ and $\rho$ are relevant coefficient, $c_{1-4}$ are constants. For each parameter, there are two portions: the nominal portion and the uncertain portion. For the nominal portions $c_{1-4}$, they can be obtained based on the relevant parameters of the previous stage. For the uncertain portions, the coefficients $\phi, \psi, \mu, \rho$ are treated as fuzzy numbers which are used to compensate for the unpredictable uncertain factors. Furthermore, $\phi, \psi$ and $\rho$ are negative, $\psi$ is positive. $\phi$ and $\psi$ are negative, which means that people will take more measures to prevent illness when the number infected cases $I$ increases. $\mu$ is positive, which means that the recovery rate will increase over time because of more medical experience. Similarly, the mortality rate will decrease over time, which means $\rho$ is

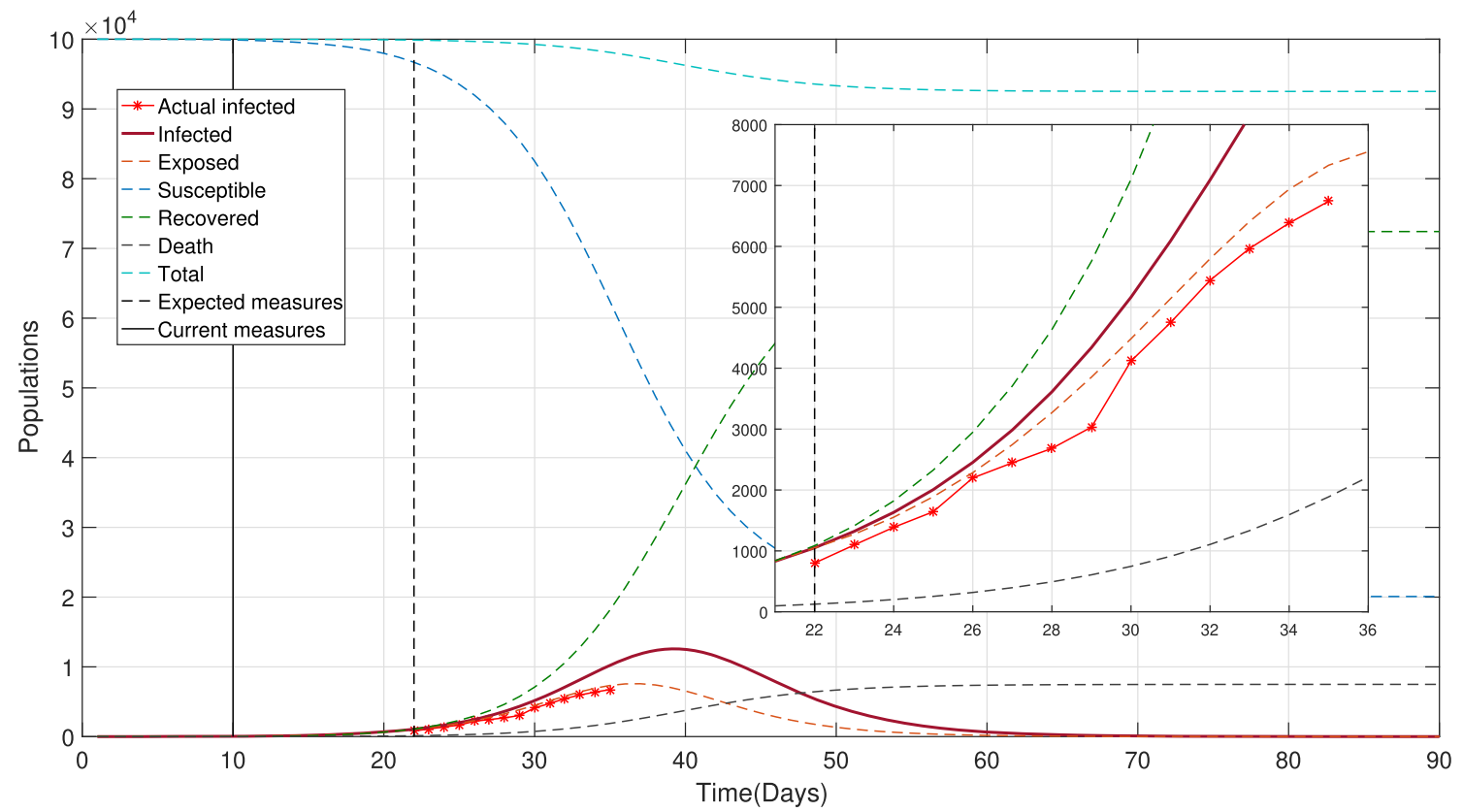

Fig. 5 Disease development based on expected measures 
negative. Hence, the parameters $r_{2}(\Delta I), b_{2}(\Delta I), y(T)$ and $u(T)$ in this the modified model is not fixed.

Figure 6 is given to illustrate the parameter design process and the differences between two models. First, the modified model is proposed with the variable parameters $r_{2}(I), b_{2}(I), y(T), u(T)$, which are regarded as the functions of $I$ and $T$. Then, the variable parameters are divided into two portions: the nominal portions $C_{1-4}$ and the uncertain portions $\phi \Delta I, \psi \Delta I, \mu T, \rho T$. The nominal portion is constant which is based on the corresponding parameter in the classical model. The uncertain portions are unknown which depends on many uncertain factors such as humidity and future policies. Hence, the coefficients in the uncertain portions are considered as fuzzy numbers and described by fuzzy sets theory. Then, the confidence theory is applied to obtain the expectation value of the coefficients.

To describe the coefficients, the fuzzy set theory and confidence index theory are adopted. The following preliminary knowledge about fuzzy arithmetic is needed before presenting the fuzzy system development [32].

(A) Fuzzy Number. Let $G$ be a fuzzy set in $\mathbf{R}$, the real number. $G$ is called fuzzy number if: (i) $G$ is normal, (ii) $G$ is convex, (iii) the support of $G$ is bounded, and (iv) all $\alpha$-cuts are closed intervals in $\mathbf{R}$. In addition, the universe of discourse of a fuzzy number is considered to be its 0 -cut.

(B) Fuzzy Arithmetic. Let $G$ and $H$ to be two fuzzy numbers and $G_{\alpha}=\left[g_{\alpha}^{-}, g_{\alpha}^{+}\right], H_{\alpha}=\left[h_{\alpha}^{-}, h_{\alpha}^{+}\right]$be their $\alpha$-cuts, $\alpha \in[0,1]$. The addition, subtraction, multiplication, and division of $G$ and $H$ are defined as follows:

$$
\begin{aligned}
& (G+H)_{\alpha}=\left[g_{\alpha}^{-}+h_{\alpha}^{-}, g_{\alpha}^{+}+h_{\alpha}^{+}\right], \\
& (G-H)_{\alpha}=\left[\min \left(g_{\alpha}^{-}-h_{\alpha}^{-}, g_{\alpha}^{+}-h_{\alpha}^{+}\right),\right. \\
& \left.\quad \max \left(g_{\alpha}^{-}-h_{\alpha}^{-}, g_{\alpha}^{+}-h_{\alpha}^{+}\right)\right], \\
& (G \cdot H)_{\alpha}=\left[\min \left(g_{\alpha}^{-} h_{\alpha}^{-}, g_{\alpha}^{-} h_{\alpha}^{+}, g_{\alpha}^{+} h_{\alpha}^{-}, g_{\alpha}^{+} h_{\alpha}^{+}\right),\right. \\
& \left.\quad \max \left(g_{\alpha}^{-} h_{\alpha}^{-}, g_{\alpha}^{-} h_{\alpha}^{+}, g_{\alpha}^{+} h_{\alpha}^{-}, g_{\alpha}^{+} h_{\alpha}^{+}\right)\right],
\end{aligned}
$$

$$
\begin{aligned}
& (G / H)_{\alpha}=\left[\min \left(g_{\alpha}^{-} / h_{\alpha}^{-}, g_{\alpha}^{-} / h_{\alpha}^{+}, g_{\alpha}^{+} / h_{\alpha}^{-}, g_{\alpha}^{+} / h_{\alpha}^{+}\right),\right. \\
& \left.\max \left(g_{\alpha}^{-} / h_{\alpha}^{-}, g_{\alpha}^{-} / h_{\alpha}^{+}, g_{\alpha}^{+} / h_{\alpha}^{-}, g_{\alpha}^{+} / h_{\alpha}^{+}\right)\right],
\end{aligned}
$$

(C) Decomposition Theorem. Define a fuzzy set $\widehat{\mathfrak{D}}_{\alpha}$ in $\mathfrak{U}$ with the membership function $\varpi_{\widehat{D}_{\alpha}}=\alpha I_{\mathfrak{D}_{\alpha}}(x)$ where $I_{\mathfrak{D}_{\alpha}}(x)=1$ if $x \in \mathfrak{D}_{\alpha}$ and $I_{\mathfrak{D}_{\alpha}}(x)=0$ if $x \in \mathfrak{U}-\mathfrak{D}_{\alpha}$. Then, the fuzzy set $\mathfrak{D}$ is obtained as

$$
\mathfrak{D}=\bigcup_{\alpha \in[,]} \widehat{\mathfrak{D}}_{\alpha}
$$

where $\bigcup$ is the union of the fuzzy sets (that is, sup over $\alpha \in[0,1]$ ). Based on these, after the operation of two fuzzy numbers via their $\alpha$-cuts, one may apply the decomposition theorem to build the membership function of the resulting fuzzy number.

Next, we assume that for each uncertainty, namely $\phi, \psi, \mu, \rho$, they lie within known fuzzy sets $K_{i}$, $i=1,2,3,4$.

$$
\begin{aligned}
& K_{1}=\left\{\left(\phi, \varpi_{1}(\phi)\right) \mid \phi \in \Omega_{1}\right\}, \\
& K_{2}=\left\{\left(\psi, \varpi_{2}(\psi)\right) \mid \psi \in \Omega_{2}\right\}, \\
& K_{3}=\left\{\left(\mu, \varpi_{3}(\mu)\right) \mid \mu \in \Omega_{3}\right\}, \\
& K_{4}=\left\{\left(\rho, \varpi_{4}(\rho)\right) \mid \rho \in \Omega_{4}\right\},
\end{aligned}
$$

where $\Omega_{i} \in \mathbf{R}$ is the known and compact universe of discourse, $\varpi_{i}: \Omega_{i} \rightarrow[0,1]$ is the membership function of $K_{i}$. In this way, the membership functions are presented based on fuzzy set theory to describe the uncertain coefficients. It means that the coefficients are fuzzy numbers.

To measure the fuzzy numbers, their expectation values are obtained based on confidence index theory. Consider the fuzzy number $k$. Its expectation value $\widehat{k}$ is used to represent the average value of the fuzzy set. The definition is given as follows: For each $\alpha \in[0,1]$, the value of the fuzzy number $k$ lies in the interval $X_{\alpha}=\left[\underline{k}_{\alpha}, \bar{k}_{\alpha}\right]$. The set $X_{\alpha}$ is also called the $k$ 's $\alpha$-cut. In addition, for all $\alpha \in[0,1]$, there exists a scalar function $f(\cdot):[0,1] \rightarrow[0,1]$, which

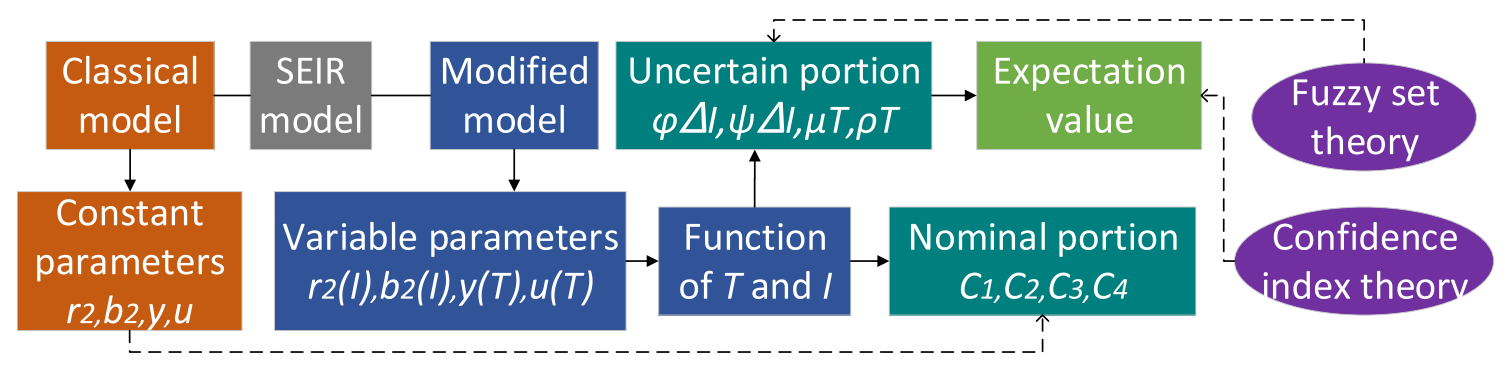

Fig. 6 Modified model and parameters design procedure 
satisfies $f(0)=1, f(1)=0 ; f(\cdot)$ is $C^{1} ; \frac{d f(\alpha)}{d \alpha}<0$. For a given $\alpha$, the value $f(\alpha)$ is called the confidence index.

Remark 3 The confidence index $f(\alpha)$ guarantees that the value of $k$ lie in the interval $\left[\underline{k}_{\alpha}, \bar{k}_{\alpha}\right]$ for any $\alpha \in[0,1]$. It can be understood as the possibility that $k$ lies in the interval $\left[\underline{k}_{\alpha}, \bar{k}_{\alpha}\right]$ is $f(\alpha)$, compared to the whole fuzzy set $X$.

Assume that the fuzzy number is "close to $k_{m}$ ". Let the lower-inverse membership function $k^{-}(\alpha)$ to describe the mapping relationship: $\alpha \rightarrow k^{-}(\alpha):[0,1] \rightarrow\left[\underline{k}_{0}, k_{m}\right]$. In the same way, the upper-inverse membership function $k^{+}(\alpha)$ is given to describe the mapping relationship: $\alpha \rightarrow k^{+}(\alpha):[0,1] \rightarrow\left[\bar{k}_{0}, k_{m}\right]$.

For the fuzzy number $k$, the confidence index $f(\alpha)$ assures that the value of $k$ lies in the interval $X_{\alpha}=\left[\underline{k}_{\alpha}, \bar{k}_{\alpha}\right]$ for any $\alpha \in[0,1]$. The lower-inverse membership function and upper-inverse membership function are also given as $k^{-}(\alpha)$ and $k^{+}(\alpha)$. Then, the expectation value $\widehat{k}$ of the fuzzy number $k$ can be obtained as

$$
\widehat{k}=\underline{k}_{0}+\int_{0}^{1}\left[k^{+}(\alpha)-k^{-}(\alpha)\right] f(\alpha) d \alpha .
$$

Remark 4 Based on the confidence index theory, the expectation value represents the average value of the fuzzy number over its membership function. It can be considered that the expectation value reflects all confidence depicted by a fuzzy set.

Now, we propose the membership functions of $k_{1-4}$ based on the actual data.

$$
\begin{gathered}
\varpi_{\phi}(v)=\left\{\begin{array}{cc}
100,00 v+3, & -3 \times 10^{-4} \leq v \leq-2 \times 10^{-4} \\
-10,000 v-1 . & -2 \times 10^{-4} \leq v \leq-1 \times 10^{-4}
\end{array}\right. \\
\varpi_{\psi}(v) \\
=\left\{\begin{array}{cc}
400,000 v+6, & -1.5 \times 10^{-5} \leq v \leq-1.25 \times 10^{-5} \\
-400,000 v-4 . & -1.25 \times 10^{-5} \leq v \leq-10^{-5}
\end{array}\right. \\
\varpi_{\mu}(v)=\left\{\begin{array}{cc}
5000 v-1, & 2 \times 10^{-4} \leq v \leq 4 \times 10^{-4} \\
-5000 v+3 . & 4 \times 10^{-4} \leq v \leq 6 \times 10^{-4}
\end{array}\right. \\
\varpi_{\rho}(v) \quad \begin{array}{cc}
100,000 v+9.5, & -9.5 \times 10^{-5} \leq v \leq-8.5 \times 10^{-5} \\
-100,000 v-7.5 . & -8.5 \times 10^{-5} \leq v \leq-7.5 \times 10^{-5}
\end{array}
\end{gathered}
$$

Remark 5 The membership functions are chosen based on the actual data. Then, the expectation value of these fuzzy coefficients can be solved based on confidence index theory. Further, some key parameters in the modified model are decided by these coefficients. Compared with the normal model with constant parameters, the modified model can reflect the actual situation more accurately due to the dynamic parameters.
The parameter $\phi$ is taken as an example to show how to obtain the expectation value of a fuzzy number by confidence index theory. Then, the fuzzy number $\phi$ lies in interval $\Phi_{\alpha}$ for $\alpha \in[0,1]$. Figure 7 is used to illustrate the concepts of membership function and confidence index.

In Fig. 7, the membership function of the fuzzy number $\phi$ is $\varpi_{\phi}(v)$. It shows that the universe of discourse of the fuzzy set $\Phi$ is the interval when $\alpha=0$. Under this circumstance, the confidence index $f(0)=1$, which means that the possibility of the value of the fuzzy number $\phi$ lying within this interval is 1 . So, we can say that the confidence index $f(\alpha)$ implies the chance that $\phi$ belongs to the interval $\left[\phi_{\alpha}, \bar{\phi}_{\alpha}\right]$. The blue lines in two partial figures represent the lower membership function and lower-inverse membership function, respectively. And the green lines represent the upper membership function and upper-inverse membership function, respectively. From this figure, the upper-inverse

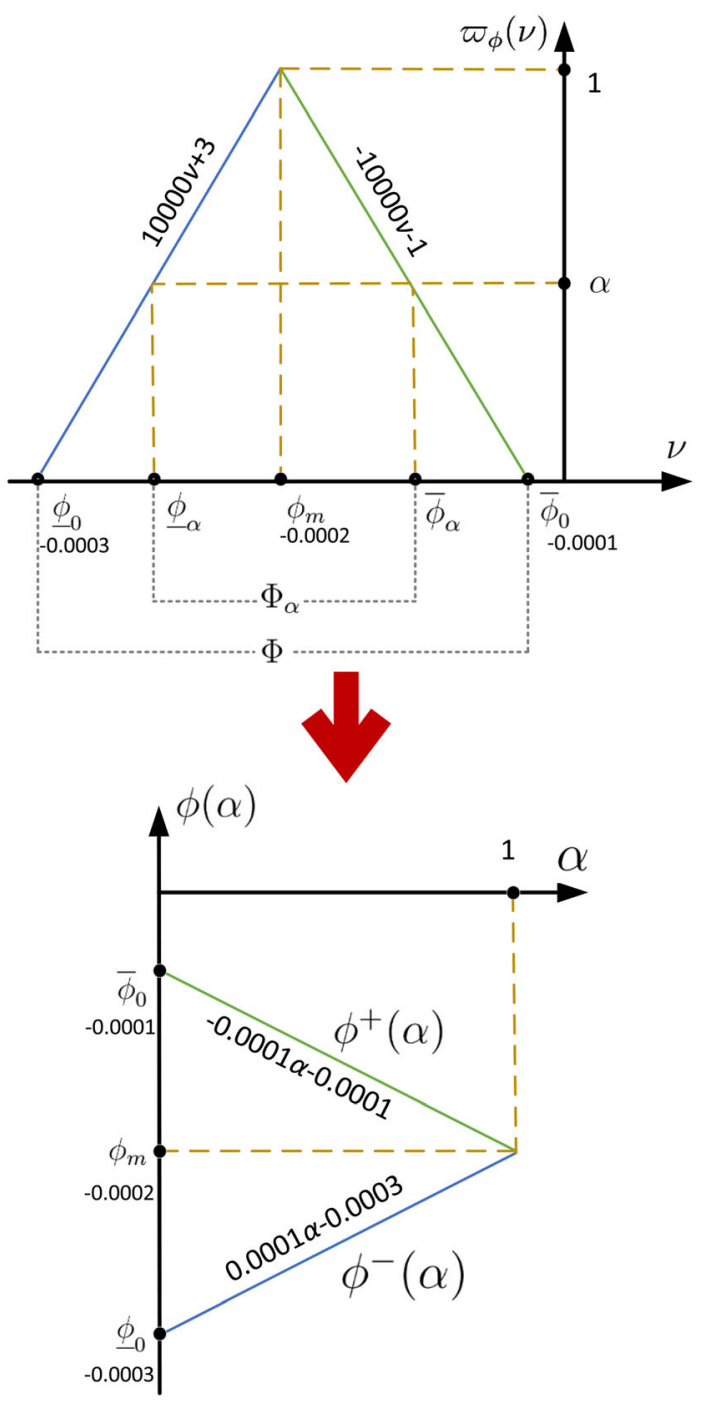

Fig. 7 Membership function of the fuzzy number $\phi$ 
membership function and lower-inverse membership function can be expressed as

$$
\begin{aligned}
& \phi^{+}(\alpha)=-0.0001 \alpha-0.0001 \quad 0 \leq \alpha \leq 1 \\
& \phi^{-}(\alpha)=0.0001 \alpha-0.0003 \quad 0 \leq \alpha \leq 1
\end{aligned}
$$

Then, based on (9), the expectation values $\widehat{\phi}, \widehat{\psi}, \widehat{\mu}, \widehat{\rho}$ can be obtained.

\section{Prediction Based on Fuzzy Parameters}

Figure 8 is used to show the development forecast of the disease with the infected-number-based and time-based parameters $r_{2}(I), b_{2}(I), y(T), u(T)$, in which the coefficients are based on fuzzy set theory and confidence index theory. The actual data from 03-23 to 04-05 were added in the revised version.

From the 1st day to the 10th day, there is no measure for this disease. The development of the disease is the same as shown in Fig. 3. On the 11th day, some measures are adopted to prevent the spread of disease. The development from day 11 to 21 is the same as shown in Fig. 4. We expect the public's attention to the disease to change as the infected number increases. People may stay at home to reduce contact with others. As a result, the parameters $r_{2}$ and $b_{2}$ will decrease. However, these two parameters may increase since the number of infected people continues to decrease at the end of this disease. Hence, the two parameters are regarded as two functions $r_{2}(I)$ and $b_{2}(I)$ after the 22 nd day. At the same time, more patients may be cured as research on the disease progresses. Thus, the parameters $y$ and $u$ are regarded as two functions $y(T)$ and $u(T)$ after the $22 \mathrm{nd}$ day. Then, considering the individual differences and many uncertainties, the coefficients in these four functions are treated as fuzzy numbers. Based on the modified model, the prediction result is closer to the actual data. For more clearly, the prediction results from 03-23 to 04-05 in the partial figures of Figs. 5 and 8 are listed in Table 3 .

From Table 3, the prediction error based on the modified model is smaller than that based on the classical model. It cannot forecast the development of this disease accurately due to the fixed parameters in the classical model and uncertain factors in society. Hence, the error becomes larger over time. In the modified model, the parameters will adjust themselves based on the actual situation. The error maintains in a small value regardless of uncertainty.

In Fig. 9, the numbers of infected cases under different situations are shown. The red, green, and blue lines are from Figs. 3, 4, and 8, which represent the numbers of infected cases without measures, only with current measures and with expected measures. The blue line shows that the disease may last for around 70 days under the current measures and the peak will appear on the 38th day (04-08). However, the "stay at home" order was revoked and the public area such as Gym was allowed to open in mid-April. Until now, the total infected cases are around 60,000, which is the same as the red line shows.

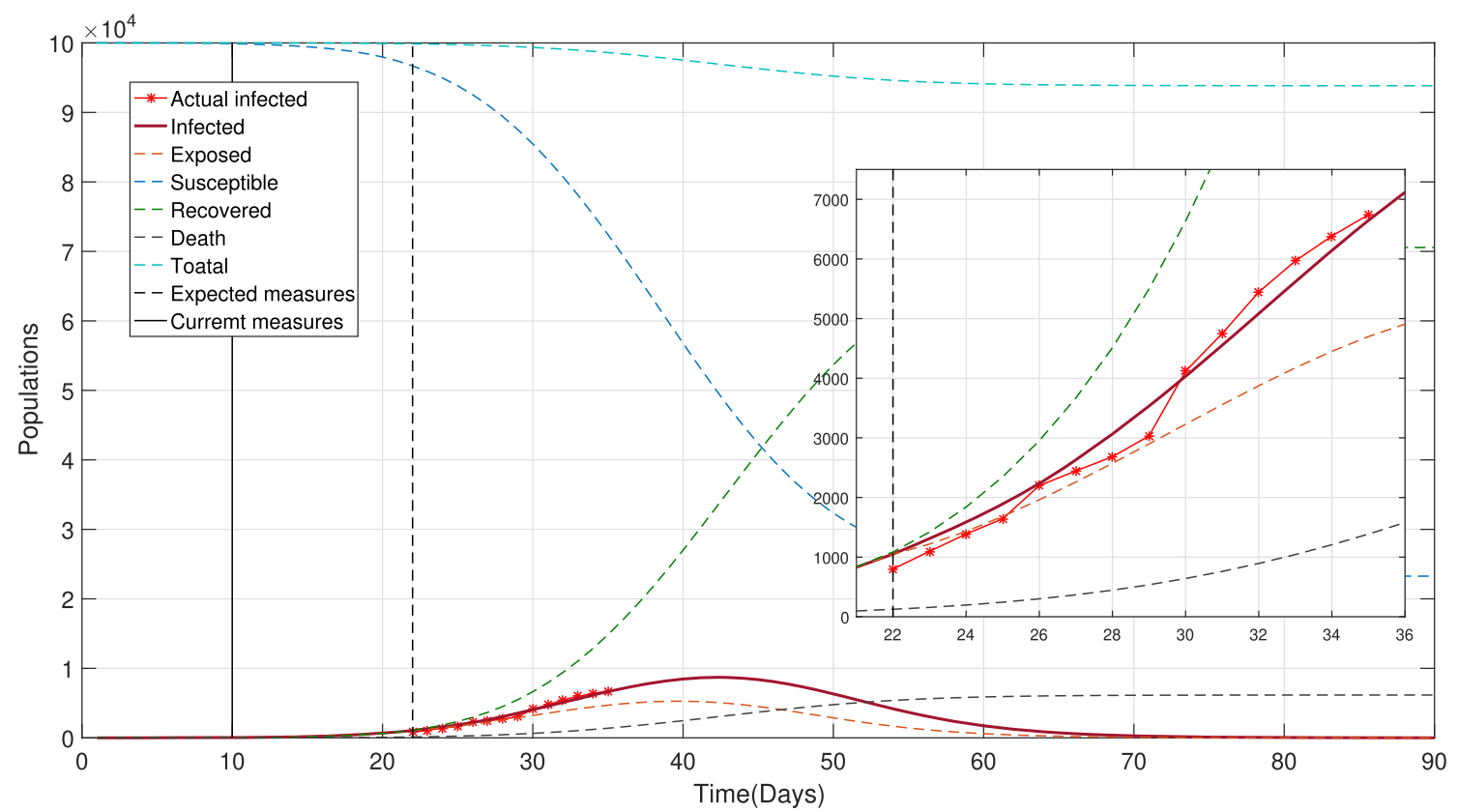

Fig. 8 Disease development with confidence index-based parameters 
Table 3 Actual data and prediction results

\begin{tabular}{|c|c|c|c|c|c|}
\hline & Actual data & $\begin{array}{l}\text { Prediction results from Fig. } 5 \\
\text { based on classical model }\end{array}$ & Error $(\%)$ & $\begin{array}{l}\text { Prediction results from Fig. } 8 \\
\text { based on modified model }\end{array}$ & Error $(\%)$ \\
\hline $03-23$ & 800 & 1051 & 31.4 & 1051 & 31.4 \\
\hline $03-24$ & 1097 & 1320 & 20.3 & 1313 & 19.7 \\
\hline $03-25$ & 1387 & 1632 & 17.7 & 1585 & 14.3 \\
\hline $03-26$ & 1643 & 2006 & 22.1 & 1890 & 15.0 \\
\hline $03-27$ & 2198 & 2453 & 11.6 & 2238 & 1.8 \\
\hline $03-28$ & 2446 & 2985 & 22.0 & 2631 & 7.6 \\
\hline $03-29$ & 2683 & 3612 & 34.6 & 3064 & 14.2 \\
\hline $03-30$ & 3032 & 4338 & 13.7 & 3533 & 16.5 \\
\hline $03-31$ & 4117 & 5166 & 25.5 & 4031 & 2.1 \\
\hline 04-01 & 4748 & 6089 & 28.2 & 4551 & 4.1 \\
\hline 04-02 & 5444 & 7071 & 29.9 & 5083 & 6.6 \\
\hline 04-03 & 5967 & 8144 & 36.5 & 5617 & 5.9 \\
\hline 04-04 & 6383 & 9206 & 44.2 & 6142 & 3.8 \\
\hline 04-08 & 6742 & 10220 & 51.6 & 6646 & 1.4 \\
\hline
\end{tabular}

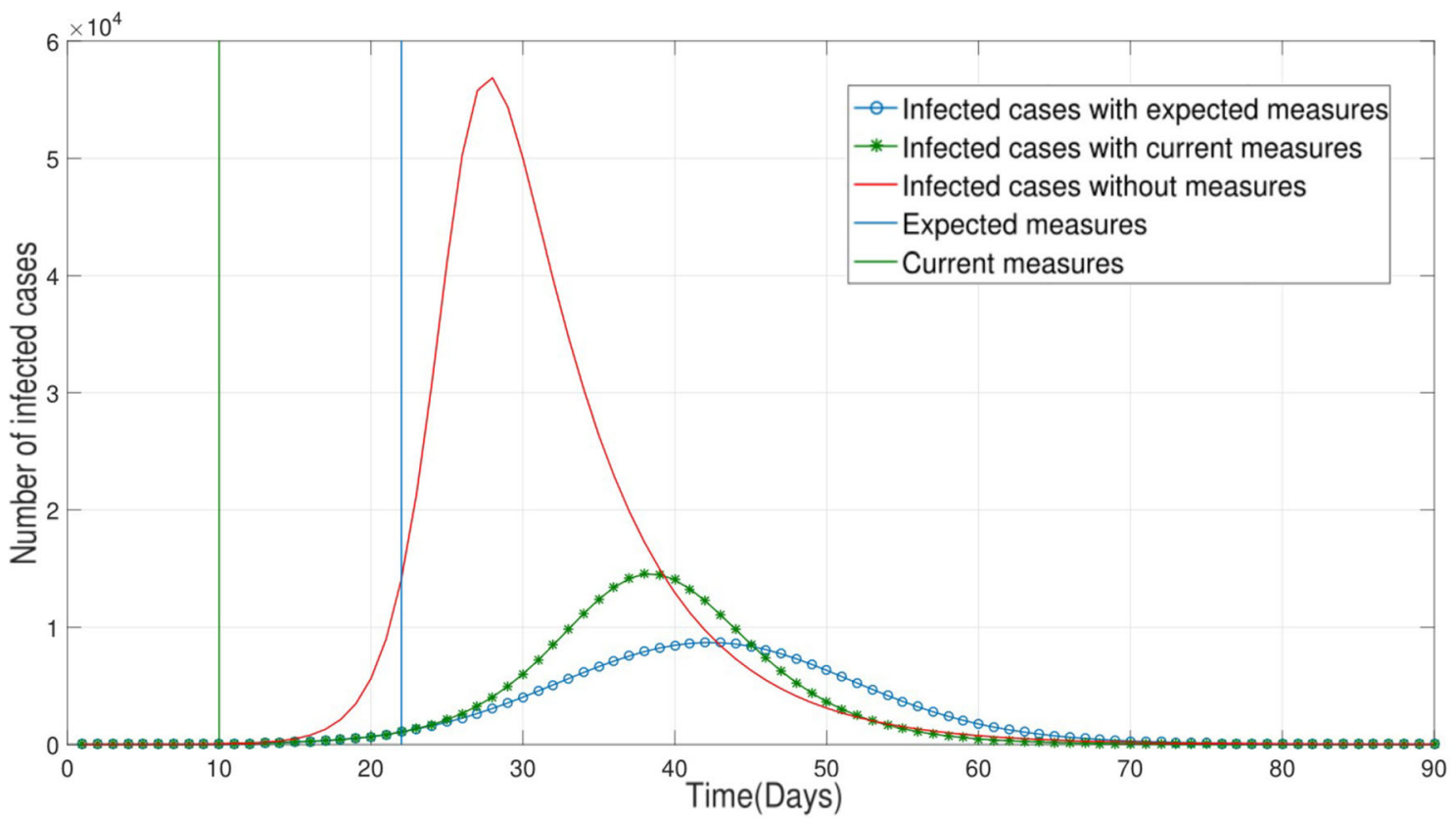

Fig. 9 Number of infected cases under different situations

\section{Conclusion}

In this paper, we analyze the coronavirus spread in the State of Georgia, USA. Based on the actual data during March 2nd and 22nd, the developments of this disease are shown under four situations which are no measures, slight measures, rigid measures with constant parameters and rigid measures with dynamic parameters. It shows that the measures such as quarantine and wearing face masks play a key role to decrease the number of death cases and infected cases by comparing the different developments of this disease.

To make the predictions fit the real situation, some key parameters in the modified SEIR model are regarded as functions of infected number and time rather than fixed constants. Then, considering the uncertainty in the actual system, we treat the relevant coefficients as fuzzy numbers. The fuzzy set theory and confidence index theory are used to identify the coefficients' expectation values. As a result, the fuzzy dynamical SEIR system is established. The 
parameters functions and related fuzzy theory which are applied in the model can not only reflect the impact of epidemic development on society but also deal with uncertainty in real society. The comparison between actual data with the prediction results under different methods shows that a smaller error can be obtained based on the proposed modified model.

Acknowledgements From this paper, we learned that this disease would have been controlled in May if the "stay at home" was still implemented. As an engineering student whose research interests are fuzzy theory and robust control, I hope to give my little contribution to help people know the importance of "stay at home" based on my specialized knowledge. This month, my son was born in Northside Hospital, Atlanta. I would like to thank the doctors and nurses who still fight on the front lines with great courage and patience. If there were not this disease, my parents would come to visit their grandson from the other side of the world and I would also prepare a family dinner for my mother's 50th birthday. I would like to commemorate the happy 50th birthday of my mother Huifang Dong and wish my son Cheney to grow healthy with this paper.

\section{References}

1. www.cdc.gov/coronavirus/2019-ncov/cases-updates/cases-in-us. html, CDC

2. Brauer, F., Castillo-Chavez, C., Castillo-Chavez, C.: Mathematical Models in Population Biology and Epidemiology. Springer, New York (2012)

3. Anderson, R.M.: The Population Dynamics of Infectious Diseases: Theory and Applications. Springer, New York (2013)

4. Hethcote, H.W.: The mathematics of infectious diseases. SIAM Rev. 42(4), 599-653 (2000)

5. McCluskey, C.C.: Complete global stability for an sir epidemic model with delay-distributed or discrete. Nonlinear Anal. 11(1), 55-59 (2010)

6. Bjørnstad, O.N., Finkenstädt, B.F., Grenfell, B.T.: Dynamics of measles epidemics: estimating scaling of transmission rates using a time series sir model. Ecol. Monogr. 72(2), 169-184 (2002)

7. Zhu, K., Ying, L.: Information source detection in the sir model: a sample-path-based approach. IEEE/ACM Trans. Netw. 24(1), 408-421 (2014)

8. Diaz, P., Constantine, P., Kalmbach, K., Jones, E., Pankavich, S.: A modified SEIR model for the spread of Ebola in western Africa and metrics for resource allocation. Appl. Math. Comput. 324, 141-155 (2018)

9. Kang, H., Yu, Y., Bao, B., Fu, X., Sun, M.: Spreading dynamics of an SEIR model with delay on scale-free networks. IEEE Trans. Netw. Sci. Eng. 7, 489-496 (2018)

10. Tang, Z., Li, X., Li, H.: Prediction of new coronavirus infection based on a modified SEIR model. medRxiv (2020)

11. Zhang, Y., Yu, X., Sun, H., Tick, G.R., Wei, W., Jin, B.: Applicability of time fractional derivative models for simulating the dynamics and mitigation scenarios of COVID-19. Chaos Solitons Fractals, 109959 (2020)
12. Piovella, N.: Analytical solution of SERI model describing the free spread of the COVID-19 pandemic. Chaos Solitons Fractals, vol. 140 (2020)

13. Yang, Y., Xu, L.: Stability of a fractional order SERI model with general incidence.' Appl. Math. Lett., vol. 105

14. Strippoli, S.: Confidence interval. Central African Journal of Medicine, vol. 45, no. 1 (1999)

15. Huang, E.J., Fang, E.X., Hanley, D.F., Rosenblum, M.: "Constructing a confidence interval for the fraction who benefit from treatment, using randomized trial data," Biometrics, vol. 75 (2019)

16. Zhang, L., Luh, P.B.: Neural network-based market clearing price prediction and confidence interval estimation with an improved extended Kalman filter method. IEEE Trans. Power Syst. 20(1), 59-66 (2005)

17. Coleman, H.W., Steele, W.G.: Experimentation, Validation, and Uncertainty Analysis for Engineers. Wiley, New York (2018)

18. Xiao, B., Yin, S.: Velocity-free fault-tolerant and uncertainty attenuation control for a class of nonlinear systems. IEEE Trans. Indust. Electron. 63(7), 4400-4411 (2016)

19. Li, C., Zhao, H., Sun, H., Chen, Y.-H.: Robust bounded control for nonlinear uncertain systems with inequality constraints. Mech. Syst. Signal Process. 140, 106665 (2020)

20. Wang, H., Bai, W.: Finite-time adaptive fault-tolerant control for strict-feedback nonlinear systems. In: 2019 Chinese Control and Decision Conference (CCDC) (2019)

21. Zadeh, L.A.: Fuzzy sets. Inform. Control 8(3), 338-353 (1965)

22. Faizi, S., Rashid, T., Sałabun, W., Zafar, S., Wkatróbski, J.: Decision making with uncertainty using hesitant fuzzy sets. Int. J. Fuzzy Syst. 20(1), 93-103 (2018)

23. Garg, H.: Linguistic pythagorean fuzzy sets and its applications in multiattribute decision-making process. Int. J. Intell. Syst. 33(6), 1234-1263 (2018)

24. Nagarajan, D., Lathamaheswari, M., Broumi, S., Kavikumar, J.: A new perspective on traffic control management using triangular interval type-2 fuzzy sets and interval neutrosophic sets. Oper. Res. Perspect. 6, 100099 (2019)

25. Xu, J., Fang, H., Zeng, F., Chen, Y.-H., Guo, H.: Robust observer design and fuzzy optimization for uncertain dynamic systems. Int. J. Fuzzy Syst. 21(5), 1511-1523 (2019)

26. Dong, F., Chen, Y.-H., Zhao, X.: Optimal design of adaptive robust control for fuzzy swarm robot systems. Int. J. Fuzzy Syst. 21(4), 1059-1072 (2019)

27. Li, C., Chen, Y.H., Sun, H., Zhao, H.: Optimal design of highorder control for fuzzy dynamical systems based on the cooperative game theory. Trans. Cybernet. 99, 1-10 (2020)

28. Yin, H., Chen, Y.-H., Yu, D.: Rendering optimal design in controlling fuzzy dynamical systems: a cooperative game approach. IEEE Trans. Indust. Inform. 15(8), 4430-4441 (2018)

29. Li, M.Y., Graef, J.R., Wang, L., Karsai, J.: Global dynamics of a SEIR model with varying total population size. Math. Biosci. 160(2), 191-213 (1999)

30. https://en.wikipedia.org/wiki/COVID-19 pandemic in Georgia (US state)

31. www.ajc.com/news/breaking-news/second-coronavirus-patientarrives-georgia-special-quarantine-site/wd3FbOD7MhZaQpxdmm RA5J/

32. Wang, L.X.: A Course in Fuzzy Systems. Prentice Hall, Upper Saddle River (1999) 


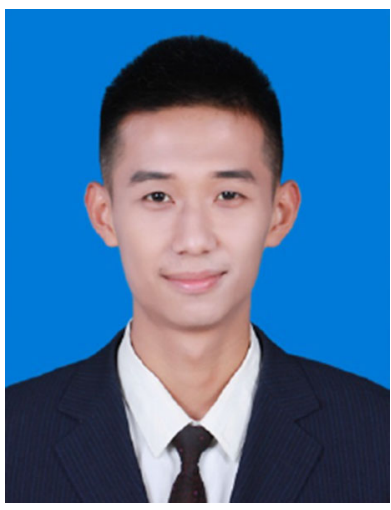

Chenming $\mathbf{L i}$ is currently pursuing the Ph.D. degreeat the School of Mechanical Engineering, Hefei Universityof Technology. He is also a visiting scholarat the George W.Woodruff School of MechanicalEngineering, Georgia Institute of Technology. Hisresearch interests include mechanical system dynamics,optimal design of robust control, adaptive robustcontrol, and fuzzy engineering.

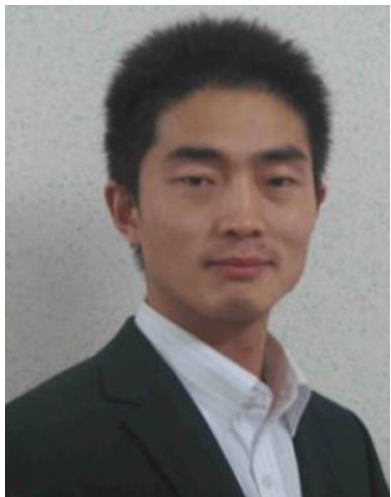

Jin Huang received his $\mathrm{Ph} . \mathrm{D}$ and B.E. degreesfrom the College of Mechanical and Vehicle Engineering,Hunan University, Changsha, China, in2012and 2006. He is also a joint Ph.D. in the GeorgeW. Woodruff School of Mechanical Engineering,Georgia Institute of Technology, Atlanta, Georgia,USA, during 2009-2011. Then he started his careeras the postdoc and assistant research professor atthe School of Software, Tsinghua University, Beijing,China, since 2013 and 2016, respectively. Hisresearch interests include artificial intelligence inIntelligent Transportation Systems, Cyber-Physical systems, automation andmechatronics, fuzzy engineering, etc.

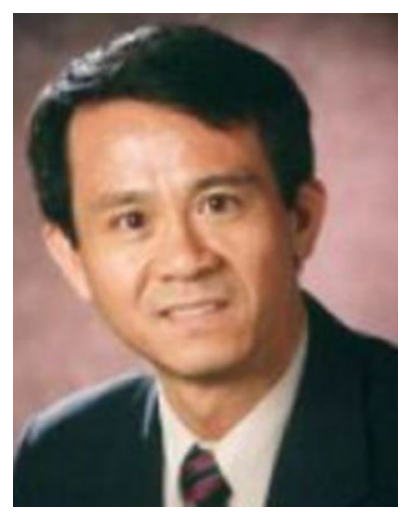

Ye-Hwa Chen received the Ph.D. degree in mechanicalengineering from the University of California,Berkeley, CA, USA, in $1985 . \mathrm{He}$ is currently a Professor with the GeorgeW.Woodruff School of Mechanical Engineering,Georgia Institute of Technology, Atlanta, GA, USA.His research interests include advanced controlmethods for mechanical systems, fuzzy engineering,adaptive robust control, and uncertainty management.

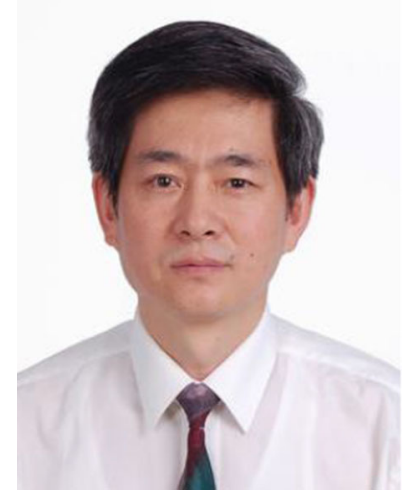

Han Zhao received the Ph.D. degree in mechanicalengineering from Aalborg University, Aalborg, Denmark,in 1990.He is currently a Professor with the School ofMechanical Engineering, Hefei University of Technology.His research interests include mechanicalsystem dynamics, vehicle system dynamics, optimalcontrol, adaptive robust control, and fuzzy engineering. 